\title{
Allometric Equations to Estimate the Aboveground Biomass of Seedling and Sapling Plants in 10 and 20 Years Old of Secondary Forests in Sarawak, Malaysia
}

\author{
Karyati ${ }^{1, *}$ Isa B. Ipor ${ }^{2}$ Ismail Jusoh ${ }^{2}$ Mohd. Effendi Wasli ${ }^{2}$ \\ ${ }^{1}$ Faculty of Forestry, University of Mulawarman, Kampus Gunung Kelua, Samarinda, East Kalimantan, 75119, \\ Indonesia. \\ ${ }^{2}$ Faculty of Resource Science and Technology, Universiti Malaysia Sarawak, 94300, Kota Samarahan, Sarawak, \\ Malaysia. \\ "Corresponding author. Email: karyati@fahutan.unmul.ac.id
}

\begin{abstract}
The seedlings and saplings plant stage determines the successional stages in the secondary forest establishment process. The estimation on aboveground biomass (AGB) of seedling and sapling plants is needed to describe undergrowth's contribution in the secondary forest. This study's objective was to develop allometric equations for accurate estimation of AGB for seedlings-saplings in 10 and 20 years old of secondary forests. The study was carried out at sites with two stages of the fallow period: lands with a fallow period of 10 and 20 years, respectively, in Sarawak, East Malaysia. The AGB data of all selected seedlings and saplings with the different species within 100 sample quadrates were used to develop allometric equations for seedlings and saplings in each study site. This study developed allometric equations to estimate AGB of seedlings-saplings (diameter at the ground surface of $<5 \mathrm{~cm}$ ), particularly in 10 and 20 years of fallow ages.
\end{abstract}

Keywords: Aboveground Biomass, Seedling, Sapling, Secondary Forest, Allometric Equation

\section{INTRODUCTION}

Tree diversity is essential to predict tree carbon storage in hyperdiverse forests [1]. The total standing aboveground biomass (AGB) of woody vegetation elements is often one of the largest carbon pools. The AGB comprises all woody stems, branches, leaves of living trees, creepers, climbers, epiphytes, and herbaceous undergrowth [2]. AGB estimation is an essential aspect of carbon stocks studies and the effects of deforestation and carbon sequestration on the global carbon balance [3]. Because direct measurement of biomass cannot be made on an entire community or population, samples must be taken from a community or population [4]. Moreover, weighing tree biomass in the field is undoubtedly the most accurate method of estimating AGB. It is still an extraordinarily timeconsuming and destructive method, generally limited to small areas and tree sample sizes [3].

An estimate of the vegetation biomass can provide information about the nutrients and carbon stored in the vegetation as a whole or the amount in specific fractions such as extractable wood [2]. Allometry is an effective method for accurately estimating trees' biomass, tree components, and stands [5]. It is hardly ever possible to measure all biomass on a sufficiently large sample area by destructive sample. Some form of allometry is used to estimate individuals' trees' biomass to an easily measured property such as its stem diameter [2]. Various dimensions and partial biomass of trees, such as bole wood, bark, branch, and foliage mass, are estimated from the diameter at breast height (DBH) by the allometric correlation method [6,7].

The allometric equation expresses the relationship between a tree's dimension or different parts of plants with the biomass $[8,9]$. Regression models are used to convert inventory data into an estimate of trees' biomass $[9,10]$. Once an allometric equation has been established for different classes of trees in vegetation, one only needs to measure DBH (or other parameters used as a basis for equation, such as height and total biomass or carbon content) to estimate the biomass of individual trees $[2,8]$. 


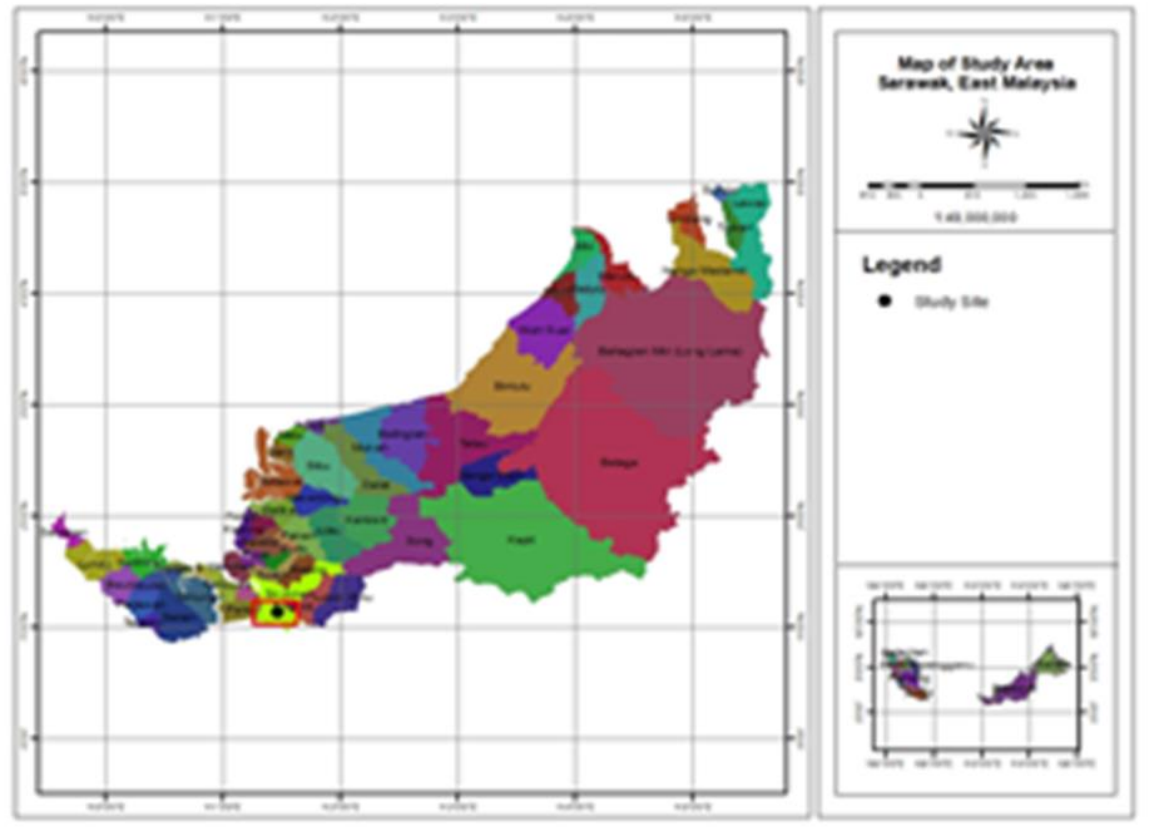

Figure 1 Map of the study area in Sabal, Sarawak, Malaysia

Because it is crucial to estimate AGB in different stage secondary forests accurately, suitable allometric equations are essential. This study's objective was to develop allometric equations for accurate estimation of AGB for seedlings-saplings in 10 and 20 years of fallow periods. Information on the study sites' dominant species and soil properties was reported by $[11,12]$. The specific selection seedlings-saplings samples were needed because mixed seedlings-saplings species characterize the secondary forests.

\section{MATERIALS AND METHODS}

\subsection{Study Sites}

The study was conducted in 10 and 20 years old of secondary forests in Sabal, Sri Aman, Sarawak, East Malaysia (figure 1). The geographic locations of these sites are $01^{\circ} 03^{\prime} 55.9^{\prime \prime} \mathrm{N} 110^{\circ} 55^{\prime} 51.4^{\prime \prime} \mathrm{E}$ and $01^{\circ} 03^{\prime} 59.3^{\prime \prime} \mathrm{N}$ $110^{\circ} 53{ }^{\prime} 34.4^{\prime \prime} \mathrm{E}$ as reported for the previous studies by $[11,12,13]$. This study was carried out for a duration of 6 months from January 2013 to July 2013.

\subsection{Data Collection}

One hundred sample quadrates of $1 \mathrm{~m} \times 1 \mathrm{~m}$ size were placed randomly in each study site for destructive sampling technique of all woody seedlings and saplings (diameter at the ground surface, Do of $<5 \mathrm{~cm}$ ). All seedlings and saplings within the sample quadrate were enumerated and identified. The different species of seedlings and saplings in every sample quadrate were selected for destructive samples. The AGB data of all selected seedlings and saplings with the other species within 100 sample quadrates were used to developed allometric equations for seedlings and saplings in each study site. Diameter at the ground surface (Do) and the total height of seedlings and saplings were measured using a digital micro caliper (Absolute Digimatic Mitutoyo) and tape, respectively. All parts of seedlingssaplings plants such as leaf and twig, branch, and stem samples were separated and weighed.

\subsection{Data Analysis}

\subsubsection{Analysis of Dry-weight in the Laboratory}

The total oven-dry weight of each seedling-sapling part was determined using the following formula $[2,9,14]$ :

$\mathrm{dw}=(\mathrm{sdw} \times \mathrm{fw}) / \mathrm{sfw}$

where: $\mathrm{dw}=$ total dry weight $(\mathrm{kg})$; sdw = dry weight of the sample $(\mathrm{g}) ; \mathrm{fw}=$ total fresh weight $(\mathrm{kg}) ; \mathrm{sfw}=$ fresh weight of the sample $(\mathrm{g})$.

\subsubsection{Tested Allometric Equations}

In the first stage of developing allometric equations for estimated AGB in the study sites, the five selected allometric equations of AGB were tested:

$$
\begin{aligned}
& y=a+b x \\
& y=a x^{b} \\
& y=a+b(\ln x) \\
& (\ln y)=a+b x \\
& (\ln y)=a+b(\ln x)
\end{aligned}
$$


where:

$\mathrm{y}=$ total dry weight or biomass of each seedling-

sapling plant part, such as stem, branch, leaf, and total aboveground biomass (TAGB) (kg)

$\mathrm{x}=$ diameter at the ground surface $(\mathrm{Do}, \mathrm{cm})$, full height $(\mathrm{H}$, meter $)$, and $(\mathrm{Do} 2 \times \mathrm{H})(\mathrm{cm} 2 \mathrm{~m})$

'a' and 'b' = coefficients estimated by regression

\subsubsection{Testing the Reliability of Model}

The allometric equation's reliability was tested based on the significant parameters (P-value) and the determination coefficient value (adjusted R2). The best regression was selected based on the goodness of fit, focusing on the suitable scatter plot, good P-value, and the high value of adjusted R2 among all tested regressions.

\section{RESULTS AND DISCUSSION}

\subsection{Selected Sample Seedlings and Saplings}

The harvested seedlings and saplings varied from 0.2 to $4.8 \mathrm{~cm}$ in Do and from 0.5 to $5.4 \mathrm{~m}$ in height in 10 years old secondary forest. The Do ranged 0.4-4.4 $\mathrm{cm}$, and height ranged $0.6-4.8 \mathrm{~m}$ for selective sample seedlings and saplings in 20 years old secondary forest. All data sets used to develop allometric equations in 10 and 20 years old of secondary forests were shown in Tables 1 and 2 .

Table 1. All data sets for develop allometric equations in 10 years old secondary forest.

\begin{tabular}{|c|c|c|c|c|c|c|c|c|}
\hline No. & Family & Species & $\begin{array}{l}\text { Do } \\
(\mathrm{cm})\end{array}$ & $\begin{array}{l}\mathrm{H} \\
(\mathrm{m})\end{array}$ & $\begin{array}{l}\text { Leaf } \\
(\mathrm{kg})\end{array}$ & $\begin{array}{c}\text { Branch } \\
(\mathrm{kg})\end{array}$ & $\begin{array}{l}\text { Stem } \\
(\mathrm{kg})\end{array}$ & $\begin{array}{c}\text { TAGB } \\
(\mathrm{kg})\end{array}$ \\
\hline 1 & Ampelidaceae & Leea indica (Burm.f.) Merr. & 1.6 & 0.8 & 0.001 & & 0.003 & 0.004 \\
\hline 2 & Annonaceae & Goniothalamus malayanus Hook. f. \& Thomson & 0.7 & 0.9 & 0.005 & & 0.006 & 0.011 \\
\hline 3 & Annonaceae & Polyalthia glauca Boerl. & 0.7 & 1.2 & 0.006 & & 0.007 & 0.013 \\
\hline 4 & Apocynaceae & A/stonia pneumatophora Backer ex Den Berger & 0.4 & 0.5 & 0.001 & & 0.001 & 0.002 \\
\hline 5 & Apocynaceae & Alstonia scholaris (L.) R. Br. & 1.9 & 1.4 & 0.005 & 0.009 & 0.033 & 0.047 \\
\hline 6 & Apocynaceae & Alstonia spatulata Blume & 3.8 & 5.4 & 0.113 & 0.154 & 0.391 & 0.658 \\
\hline 7 & Apocynaceae & Tabernaemontana sp. & 0.7 & 0.5 & 0.013 & & 0.004 & 0.016 \\
\hline 8 & Asteraceae & Vernonia arborea Buch. Ham. & 0.6 & 0.8 & 0.003 & & 0.003 & 0.006 \\
\hline 9 & Burseraceae & Dacryodes rostrata (Blume) H.J. Lam & 0.9 & 1.0 & 0.013 & 0.006 & 0.010 & 0.030 \\
\hline 10 & Burseraceae & Santiria rubiginosa Blume & 1.0 & 1.8 & 0.012 & 0.014 & 0.036 & 0.062 \\
\hline 11 & Burseraceae & Santiria tomentosa Blume & 0.5 & 0.7 & 0.004 & & 0.003 & 0.007 \\
\hline 12 & Clusiaceae & Cratoxylum glaucum Korth. & 1.0 & 1.4 & 0.019 & 0.008 & 0.017 & 0.045 \\
\hline 13 & Dilleniaceae & Dillenia excelsa Martelli & 1.4 & 2.9 & 0.027 & & 0.044 & 0.072 \\
\hline 14 & Dilleniaceae & Dillenia pulchella Gilg & 1.4 & 2.4 & 0.012 & & 0.086 & 0.098 \\
\hline 15 & Dilleniaceae & Dillenia suffruticosa Martelli & 1.4 & 1.3 & 0.023 & & 0.025 & 0.047 \\
\hline 16 & Dipterocarpaceae & Hopea beccariana Burck & 0.5 & 0.9 & 0.006 & & 0.006 & 0.011 \\
\hline 17 & Dipterocarpaceae & Shorea macrophylla (de Vriese) P.S. Ashton & 1.5 & 1.6 & 0.054 & 0.012 & 0.031 & 0.097 \\
\hline 18 & Dipterocarpaceae & Shorea palembanica Miq. & 1.3 & 1.6 & 0.030 & 0.011 & 0.025 & 0.066 \\
\hline 19 & Dipterocarpaceae & Shorea parvifolia Dyer & 0.5 & 0.6 & 0.001 & & 0.001 & 0.003 \\
\hline 20 & Dipterocarpaceae & Shorea sp. & 0.4 & 0.5 & 0.002 & & 0.002 & 0.004 \\
\hline 21 & Elaeocarpaceae & Elaeocarpus beccarii Aug. DC. & 2.0 & 2.7 & 0.019 & 0.027 & 0.089 & 0.136 \\
\hline 22 & Elaeocarpaceae & Elaeocarpus stipularis Blume & 1.9 & 1.2 & 0.022 & 0.007 & 0.013 & 0.043 \\
\hline 23 & Euphorbiaceae & Agrostistachys longifolia Benth. ex Hook. f. & 0.7 & 1.0 & 0.014 & & 0.008 & 0.022 \\
\hline 24 & Euphorbiaceae & Antidesma neurocarpum Miq. & 1.3 & 2.1 & 0.002 & 0.019 & 0.052 & 0.072 \\
\hline 25 & Euphorbiaceae & Aporosa sp. & 1.4 & 1.3 & 0.023 & 0.016 & 0.032 & 0.071 \\
\hline 26 & Euphorbiaceae & Baccaurea macrocarpa Mull. Arg. & 1.5 & 1.5 & 0.030 & 0.013 & 0.031 & 0.073 \\
\hline 27 & Euphorbiaceae & Cleistanthus sp. & 1.4 & 1.3 & 0.013 & & 0.020 & 0.033 \\
\hline 28 & Euphorbiaceae & Endospermum diadenum (Miq.) Airy Shaw & 0.5 & 0.6 & 0.002 & & 0.003 & 0.005 \\
\hline
\end{tabular}




\begin{tabular}{|c|c|c|c|c|c|c|c|c|}
\hline 29 & Euphorbiaceae & Macaranga beccariana Merr. & 4.8 & 3.6 & 0.111 & 0.056 & 0.319 & 0.487 \\
\hline 30 & Euphorbiaceae & Macaranga caladifolia Becc. & 0.8 & 1.8 & 0.002 & & 0.013 & 0.016 \\
\hline 31 & Euphorbiaceae & Macaranga gigantea Mull. Arg. & 1.5 & 1.2 & 0.007 & & 0.005 & 0.012 \\
\hline 32 & Euphorbiaceae & Mallotus macrostachyus Mull. Arg. & 1.4 & 2.7 & 0.008 & & 0.063 & 0.071 \\
\hline 33 & Fabaceae & Sindora beccariana Backer ex de Wit & 1.0 & 1.4 & 0.009 & 0.008 & 0.024 & 0.041 \\
\hline 34 & Fabaceae & Uraria crinita Desv. & 1.0 & 1.2 & 0.008 & 0.003 & 0.010 & 0.021 \\
\hline 35 & Fagaceae & Lithocarpus sp. & 1.1 & 2.0 & 0.030 & 0.018 & 0.038 & 0.086 \\
\hline 36 & Lauraceae & Beilschmiedia sp. & 1.7 & 3.4 & 0.061 & 0.039 & 0.134 & 0.233 \\
\hline 37 & Lauraceae & Litsea costalis (Nees) Kosterm. var. nidularis Gamble & 0.2 & 1.6 & 0.015 & & 0.013 & 0.028 \\
\hline 38 & Lauraceae & Litsea elliptica Blume & 0.7 & 1.1 & 0.004 & & 0.011 & 0.015 \\
\hline 39 & Loganiaceae & Fagraea resinosa Leenh. & 2.3 & 3.2 & 0.102 & 0.131 & 0.162 & 0.395 \\
\hline 40 & Loganiaceae & Norrisia malaccensis Gardn. & 1.1 & 1.4 & 0.002 & & 0.032 & 0.034 \\
\hline 41 & Melastomataceae & Blastus borneensis Cogn. ex Boerl. & 1.3 & 1.5 & 0.010 & & 0.052 & 0.062 \\
\hline 42 & Melastomataceae & Medinilla sp. & 0.8 & 1.4 & 0.024 & & 0.016 & 0.040 \\
\hline 43 & Melastomataceae & Pternandra multiflora Cogn. & 0.6 & 0.8 & 0.004 & & 0.008 & 0.012 \\
\hline 44 & Moraceae & Artocarpus kemando Miq. & 0.9 & 1.2 & 0.010 & 0.003 & 0.008 & 0.022 \\
\hline 45 & Moraceae & Ficus aurata Miq. & 0.9 & 1.4 & 0.008 & & 0.011 & 0.019 \\
\hline 46 & Moraceae & Ficus condensa King & 0.4 & 0.6 & 0.002 & & 0.002 & 0.004 \\
\hline 47 & Moraceae & Ficus geocharis Corner. & 2.4 & 3.0 & 0.020 & 0.031 & 0.088 & 0.139 \\
\hline 48 & Moraceae & Ficus sp. & 0.6 & 0.8 & 0.004 & & 0.005 & 0.009 \\
\hline 49 & Myristicaceae & Knema intermedia Warb. & 2.5 & 2.8 & 0.218 & 0.086 & 0.234 & 0.538 \\
\hline 50 & Myrsinaceae & Ardisia sp. & 0.9 & 1.3 & 0.013 & & 0.015 & 0.029 \\
\hline 51 & Myrtaceae & Syzygium arcuatinervum (Merr.) Craven \& Briffin & 0.3 & 0.8 & 0.001 & & 0.002 & 0.003 \\
\hline 52 & Myrtaceae & Whiteodendron moultonianum (W.W.Sm.) Steenis & 1.0 & 1.2 & 0.013 & 0.014 & 0.021 & 0.047 \\
\hline 53 & Polygalaceae & Xanthophyllum flavescens Roxb. & 0.9 & 1.2 & 0.011 & & 0.017 & 0.028 \\
\hline 54 & Rosaceae & Prunus arborea (Blume) Kalkman & 1.2 & 1.7 & 0.054 & 0.024 & 0.035 & 0.113 \\
\hline 55 & Rosaceae & Prunus beccarii (Ridl.) Kalkman & 1.4 & 1.2 & 0.010 & 0.015 & 0.103 & 0.128 \\
\hline 56 & Rubiaceae & Canthium didymum Gaertn. & 1.9 & 3.1 & 0.076 & 0.042 & 0.164 & 0.282 \\
\hline 57 & Rubiaceae & Gardenia resinifera Korth. & 0.7 & 0.8 & 0.007 & & 0.009 & 0.016 \\
\hline 58 & Rubiaceae & Nauclea subdita Merr. & 0.8 & 0.6 & 0.016 & & 0.006 & 0.022 \\
\hline 59 & Rubiaceae & Tarenna fragrans Koord. \& Valeton & 1.0 & 1.2 & 0.012 & 0.009 & 0.013 & 0.034 \\
\hline 60 & Rutaceae & Euodia glabra (BI.) BI. & 1.6 & 1.5 & 0.007 & 0.004 & 0.043 & 0.054 \\
\hline \multirow[t]{5}{*}{61} & Verbenaceae & Vitex pubescens Vahl. & 1.1 & 1.8 & 0.011 & & 0.035 & 0.046 \\
\hline & & Total & 73.6 & 94.4 & 1.367 & 0.779 & 2.692 & 4.838 \\
\hline & & Average & 1.2 & 1.5 & 0.022 & 0.029 & 0.044 & 0.079 \\
\hline & & Minimum & 0.2 & 0.5 & 0.001 & 0.003 & 0.001 & 0.002 \\
\hline & & Maximum & 4.8 & 5.4 & 0.218 & 0.154 & 0.391 & 0.658 \\
\hline
\end{tabular}

Note: $\mathrm{Do}=$ diameter at ground surface; $\mathrm{H}=$ total height; $\mathrm{TAGB}=$ total above ground biomass .

There were 61 species of 45 genera of 24 families selected in 10 years old secondary forest. The dry weight range was $0.001-0.218 \mathrm{~kg}$ for leaf, $0.003-0.154$ $\mathrm{kg}$ for branch, $0.001-0.391 \mathrm{~kg}$ for the stem, and 0.002$0.658 \mathrm{~kg}$ for TAGB in this site. Out of 61 samples, 34 samples for both seedlings and saplings were without dry branch weight (Table 1). In 20 years of secondary forest, 65 species of seedlings and saplings belonged to 45 genera, and 30 families were encountered. The dry weight varied from 0.001 to $0.336 \mathrm{~kg}$ for leaf, 0.003 to $0.258 \mathrm{~kg}$ for branch, 0.002 to $0.537 \mathrm{~kg}$ for stem, and 0.007 to $0.979 \mathrm{~kg}$ for TAGB, respectively. Twentyseven of 65 sample plants did not have a branch yet, as presented in Table 2. 
Table 2. All data sets for develop allometric equations in 20 years old secondary forest.

\begin{tabular}{|c|c|c|c|c|c|c|c|c|}
\hline No. & Family & Species & $\begin{array}{c}\text { Do } \\
(\mathrm{cm})\end{array}$ & $\begin{array}{c}\mathrm{H} \\
(\mathrm{m})\end{array}$ & $\begin{array}{l}\text { Leaf } \\
(\mathrm{kg})\end{array}$ & $\begin{array}{c}\text { Branch } \\
(\mathrm{kg})\end{array}$ & $\begin{array}{l}\text { Stem } \\
(\mathrm{kg})\end{array}$ & $\begin{array}{l}\text { TAGB } \\
(\mathrm{kg})\end{array}$ \\
\hline 1 & Ampelidaceae & Leea indica (Burm.f.) Merr. & 0.4 & 0.8 & 0.005 & & 0.002 & 0.007 \\
\hline 2 & Anisophylleaceae & Anisophyllea disticha Baill. & 1.4 & 1.0 & 0.012 & 0.013 & 0.031 & 0.056 \\
\hline 3 & Annonaceae & Goniothalamus velutinus Airy Shaw & 2.1 & 1.9 & 0.031 & 0.032 & 0.062 & 0.125 \\
\hline 4 & Annonaceae & Monocarpia sp. & 1.7 & 1.7 & 0.026 & 0.032 & 0.051 & 0.110 \\
\hline 5 & Annonaceae & Polyalthia sp. & 0.6 & 1.0 & 0.011 & & 0.010 & 0.022 \\
\hline 6 & Apocynaceae & Alstonia spatulata Blume & 0.6 & 1.0 & 0.001 & & 0.008 & 0.009 \\
\hline 7 & Apocynaceae & Tabernaemontana sp. & 1.7 & 2.1 & 0.041 & 0.025 & 0.066 & 0.132 \\
\hline 8 & Burseraceae & Santiria rubiginosa Blume & 1.2 & 2.5 & 0.076 & 0.023 & 0.068 & 0.167 \\
\hline 9 & Celastraceae & Bhesa paniculata Arn. & 1.4 & 1.3 & 0.008 & 0.006 & 0.054 & 0.067 \\
\hline 10 & Clusiaceae & Cratoxylum arborescens Blume. & 1.9 & 2.3 & 0.015 & 0.024 & 0.115 & 0.153 \\
\hline 11 & Clusiaceae & Cratoxylum formosum Benth. \& Hook. f. ex Dyer & 0.7 & 1.0 & 0.005 & & 0.004 & 0.010 \\
\hline 12 & Clusiaceae & Garcinia sp. & 1.4 & 1.3 & 0.016 & 0.020 & 0.050 & 0.086 \\
\hline 13 & Dilleniaceae & Dillenia suffruticosa Martelli & 2.4 & 2.7 & 0.109 & 0.054 & 0.175 & 0.339 \\
\hline 14 & Elaeocarpaceae & Elaeocarpus beccarii Aug. DC. & 1.2 & 2.3 & 0.031 & 0.021 & 0.047 & 0.099 \\
\hline 15 & Elaeocarpaceae & Elaeocarpus stipularis Blume & 0.6 & 1.0 & 0.005 & & 0.005 & 0.011 \\
\hline 16 & Euphorbiaceae & Antidesma neurocarpum Miq. & 1.1 & 1.2 & 0.011 & 0.003 & 0.025 & 0.040 \\
\hline 17 & Euphorbiaceae & Endospermum diadenum (Miq.) Airy Shaw & 0.8 & 1.2 & 0.003 & & 0.011 & 0.013 \\
\hline 18 & Euphorbiaceae & Macaranga beccariana Merr. & 2.2 & 3.0 & 0.070 & & 0.103 & 0.173 \\
\hline 19 & Euphorbiaceae & Macaranga gigantea Mull. Arg. & 3.2 & 4.8 & 0.115 & 0.258 & 0.278 & 0.651 \\
\hline 20 & Euphorbiaceae & Mallotus macrostachyus Mull. Arg. & 1.1 & 1.5 & 0.017 & & 0.023 & 0.040 \\
\hline 21 & Fabaceae & Fordia sp. & 1.1 & 1.6 & 0.016 & 0.010 & 0.039 & 0.064 \\
\hline 22 & Fagaceae & Lithocarpus sp. & 1.4 & 1.9 & 0.026 & 0.013 & 0.063 & 0.102 \\
\hline 23 & Ixonanthaceae & Ixonanthes reticulata Jack & 0.7 & 1.0 & 0.012 & & 0.010 & 0.022 \\
\hline 24 & Lauraceae & Actinodaphne sp. & 1.1 & 1.5 & 0.007 & 0.016 & 0.023 & 0.045 \\
\hline 25 & Lauraceae & Beilschmiedia endiandraefolia Kosterm. & 0.9 & 1.1 & 0.002 & & 0.014 & 0.016 \\
\hline 26 & Lauraceae & Litsea costalis (Nees) Kosterm. var. nidularis Gamble & 1.3 & 1.4 & 0.041 & 0.009 & 0.023 & 0.073 \\
\hline 27 & Lauraceae & Litsea crassifolia Boerl. & 1.0 & 0.8 & 0.006 & 0.003 & 0.006 & 0.015 \\
\hline 28 & Lauraceae & Litsea elliptica Blume & 2.6 & 3.2 & 0.144 & 0.084 & 0.263 & 0.491 \\
\hline 29 & Lauraceae & Litsea nidularis Gamble & 0.8 & 1.3 & 0.015 & & 0.019 & 0.033 \\
\hline 30 & Lauraceae & Litsea oppositifolia (BI.) Vill. & 0.9 & 0.9 & 0.008 & & 0.007 & 0.015 \\
\hline 31 & Loganiaceae & Norrisia malaccensis Gardn. & 2.6 & 2.7 & 0.063 & 0.056 & 0.121 & 0.240 \\
\hline 32 & Melastomataceae & Pternandra coerulescens Jack & 1.7 & 3.6 & 0.063 & 0.049 & 0.121 & 0.233 \\
\hline 33 & Moraceae & Artocarpus dadak Miq. & 1.4 & 1.8 & 0.033 & 0.029 & 0.035 & 0.097 \\
\hline 34 & Moraceae & Artocarpus elasticus Reinw. & 0.7 & 1.2 & 0.008 & & 0.006 & 0.015 \\
\hline 35 & Moraceae & Artocarpus integer (Thunb.) Merr. & 0.8 & 1.6 & 0.007 & & 0.018 & 0.025 \\
\hline 36 & Moraceae & Artocarpus kemando Miq. & 0.9 & 0.9 & 0.001 & & 0.009 & 0.010 \\
\hline 37 & Moraceae & Artocarpus nitidus Trecul & 1.5 & 3.0 & 0.047 & 0.030 & 0.053 & 0.130 \\
\hline 38 & Moraceae & Artocarpus odoratissimus Blanco & 0.8 & 1.7 & 0.013 & & 0.015 & 0.028 \\
\hline 39 & Moraceae & Ficus aurata Miq. & 4.4 & 4.3 & 0.153 & 0.192 & 0.396 & 0.741 \\
\hline 40 & Moraceae & Ficus condensa King & 0.8 & 0.8 & 0.003 & & 0.015 & 0.018 \\
\hline 41 & Moraceae & Ficus geocharis Corner & 2.5 & 3.3 & 0.121 & 0.095 & 0.285 & 0.501 \\
\hline
\end{tabular}




\begin{tabular}{|c|c|c|c|c|c|c|c|c|}
\hline 42 & Moraceae & Ficus beccarii King. & 2.6 & 4.0 & 0.054 & 0.083 & 0.332 & 0.469 \\
\hline 43 & Moraceae & Ficus sp. & 1.2 & 2.5 & 0.015 & 0.020 & 0.032 & 0.067 \\
\hline 44 & Myristicaceae & Horsfieldia grandis Warb. & 1.1 & 1.0 & 0.013 & & 0.012 & 0.024 \\
\hline 45 & Myrtaceae & Syzygium polyanthum Walp. & 1.0 & 0.9 & 0.008 & & 0.008 & 0.016 \\
\hline 46 & Polygalaceae & Xanthophyllum affine Korth. ex Miq. & 1.0 & 1.2 & 0.008 & 0.005 & 0.012 & 0.025 \\
\hline 47 & Polygalaceae & Xantophyllum ferrugineum Van der Meijden & 1.0 & 1.5 & 0.013 & & 0.019 & 0.032 \\
\hline 48 & Polygalaceae & Xantophyllum flavescens Roxb. & 1.1 & 1.5 & 0.011 & 0.006 & 0.018 & 0.035 \\
\hline 49 & Proteaceae & Heliciopsis percoriacea R.C.K. Chung & 2.2 & 2.5 & 0.071 & 0.043 & 0.152 & 0.266 \\
\hline 50 & Rosaceae & Prunus arborea (Blume) Kalkman & 0.6 & 0.7 & 0.003 & & 0.006 & 0.008 \\
\hline 51 & Rubiaceae & Gardenia resinifera Korth. & 1.5 & 1.2 & 0.041 & & 0.033 & 0.073 \\
\hline 52 & Rubiaceae & Nauclea subdita Merr. & 1.6 & 0.7 & 0.045 & 0.023 & 0.049 & 0.117 \\
\hline 53 & Rubiaceae & Tarenna fragrans Koord. \& Valeton & 1.0 & 1.2 & 0.013 & & 0.023 & 0.036 \\
\hline 54 & Sapindaceae & Lepisanthes sp. & 1.4 & 1.4 & 0.007 & & 0.093 & 0.099 \\
\hline 55 & Sapindaceae & Nephelium cuspidatum Blume & 1.2 & 0.7 & 0.002 & & 0.008 & 0.010 \\
\hline 56 & Sapotaceae & Palaquium decurrens H.J. Lam & 2.3 & 3.1 & 0.130 & 0.060 & 0.210 & 0.400 \\
\hline 57 & Sapotaceae & Palaquium gutta Burck & 1.3 & 1.7 & 0.067 & 0.021 & 0.039 & 0.128 \\
\hline 58 & Sterculiaceae & Commersonia bartramia (L.) Merr. & 0.8 & 1.1 & 0.006 & & 0.004 & 0.010 \\
\hline 59 & Theaceae & Adinandra dumosa Jack & 0.5 & 0.6 & 0.006 & & 0.011 & 0.017 \\
\hline 60 & Thymelaeaceae & Gonystylus costalis Airy Shaw & 1.4 & 1.4 & 0.012 & 0.005 & 0.025 & 0.041 \\
\hline 61 & Thymelaeaceae & Gonystylus sp. & 3.0 & 4.2 & 0.336 & 0.105 & 0.537 & 0.979 \\
\hline 62 & Tiliaceae & Brownlowia havilandii Stapf & 1.4 & 1.0 & 0.013 & 0.010 & 0.012 & 0.034 \\
\hline 63 & Tiliaceae & Grewia laevigata Vahl & 2.0 & 1.6 & 0.018 & 0.016 & 0.023 & 0.058 \\
\hline 64 & Tiliaceae & Pentace sp. & 1.1 & 1.5 & 0.023 & 0.011 & 0.017 & 0.051 \\
\hline \multirow[t]{5}{*}{65} & Ulmaceae & Gironniera nervosa Planch. & 0.8 & 1.3 & 0.011 & 0.004 & 0.014 & 0.030 \\
\hline & & Total & 91.4 & 113.7 & 2.322 & 1.511 & 4.418 & 8.251 \\
\hline & & Average & 1.4 & 1.7 & 0.036 & 0.040 & 0.068 & 0.127 \\
\hline & & Minimum & 0.4 & 0.6 & 0.001 & 0.003 & 0.002 & 0.007 \\
\hline & & Maximum & 4.4 & 4.8 & 0.336 & 0.258 & 0.537 & 0.979 \\
\hline
\end{tabular}

Note: $\mathrm{Do}=$ diameter at ground surface; $\mathrm{H}=$ total height; $\mathrm{TAGB}=$ total above-ground biomass

\subsection{The Best Selected Allometric Equations for Above Ground Biomass (AGB) of Seedlings- Saplings}

The regression analysis results for predicting plant part biomass of subject seedlings and saplings from diameter at the ground surface (Do) and total height $(\mathrm{H})$ using all studied individuals' data are shown in Table 3. From all tested regression, the best selected allometric equations to estimate seedlings and saplings were dominated by the log-linear model $(\ln \mathrm{y}=\mathrm{a}+\mathrm{b} \ln \mathrm{x}) "(8$ and 10 proposed equations in 10 and 20 years old secondary forests). These equations were the best-fitting model to relate dependent variables (leaf, branch, stem, and AGB) and independent variables (Do, (Do2 $\times \mathrm{H})$, and $\mathrm{H}$ ) for the seedlings-saplings stage. However, the result did not propose the best equations for the relationship between dry leaf biomass of seedlingsaplings and plant dimensions in 10 years old secondary forest. Among all five tested allometric equations, only two allometric equations were proposed following exponential models $(\mathrm{y}=\mathrm{a} \quad \mathrm{x} \quad \mathrm{b})$. After shifting cultivation, the allometric equations for different ages of secondary forests in fallow lands, such as 10 and 20 years fallow periods, are still rare available. Several allometric equations of secondary forests were reported by $[3,15][16,17,18]$. When no specific allometric equations estimate AGB of seedlings-saplings at a different age, secondary forests are available. These proposed equations may be used to estimate AGB at different stages of fallow periods. In addition, most previous reported allometric equations were for the trees stage. This study proposed allometric equations to estimate AGB of seedlings-saplings (Do of $<5 \mathrm{~cm}$ ), particularly in 10 and 20 years of fallow ages. The developed allometric equations were suitable for 10 and 20 years of secondary forests because the selected 
samples in the destructive method were based on the representative species.

The amount of dry biomass was influenced by the number of individuals. At the early stage of secondary forests, the occurrence of seedlings and saplings was dominant and abundant. The seedlings and saplings stage was abundant as far as the gap was available. When forests reached maturity and big trees began dominating, light availability was limited in the forest floor, caused the seedlings and saplings to decrease while increasing the forest. As [11] and [19] reported, the number of plant seedlings and saplings decreased in secondary forests with increasing fallow periods. The late pioneer and secondary species were dominant in the ten and 20-year-old secondary forests [13]. Seedling height and biomass growth varied significantly amongst the species [20]. Significant changes occur when many dominant trees senesce at the same time, creating significant gaps and giving an opening to species found at the earlier stages of succession. Replacement of canopy dominants in different age species will occur without substantial disruption of the forests' structure and biomass [21].

\section{CONCLUSION}

We conclude that the best selected allometric equations to estimate seedlings and saplings were dominated by the $\log$-linear model $(\ln \mathrm{y}=\mathrm{a}+\mathrm{b} \ln \mathrm{x})$. This study's findings propose an allometric equation of AGB in 10 and 20 years old of secondary forests under similar parent materials and land-use history (slash and burn after shifting cultivation).

\section{ACKNOWLEDGMENTS}

We thank Mr. Hidir Marzuki, Mr. Sekudan Tedong, Mr. Salim Arip, and Mr. Muhd Najib Fardos for their kind support in the fieldwork.

Table 3. The best selected allometric equations for predicting plant part biomass of subject seedlings-saplings (Do of $<5 \mathrm{~cm})$ in the study sites.

\begin{tabular}{|c|c|c|c|c|}
\hline Dependent variable $(\mathrm{y})$ & $\begin{array}{l}\text { Independent } \\
\text { variable }(x)\end{array}$ & Equation & $P$-value & Adjusted $R^{2}$ \\
\hline \multicolumn{5}{|c|}{ Ten years old secondary forest } \\
\hline \multirow[t]{2}{*}{ Branch dry biomass (kg) } & $\left(\mathrm{Do}^{2} \times \mathrm{H}\right)\left(\mathrm{cm}^{2} \mathrm{~m}\right)$ & $\ln (y)=0.6720 \times \ln (x)-5.060$ & $<0.001$ & 0.67 \\
\hline & $\mathrm{H}(\mathrm{m})$ & $\ln (y)=2.0164 \times \ln (x)-5.314$ & $<0.001$ & 0.76 \\
\hline \multirow[t]{3}{*}{ Stem dry biomass $(\mathrm{kg})$} & Do $(\mathrm{cm})$ & $\ln (y)=1.8545 \times \ln (x)-4.067$ & $<0.001$ & 0.64 \\
\hline & $\left(\mathrm{Do}^{2} \times \mathrm{H}\right)\left(\mathrm{cm}^{2} \mathrm{~m}\right)$ & $\ln (y)=0.7532 \times \ln (x)-4.280$ & $<0.001$ & 0.80 \\
\hline & $\mathrm{H}(\mathrm{m})$ & $\ln (y)=2.3739 \times \ln (x)-4.727$ & $<0.001$ & 0.85 \\
\hline \multirow[t]{3}{*}{ Aboveground biomass (kg) } & Do $(\mathrm{cm})$ & $\ln (y)=1.7911 x \ln (x)-3.425$ & $<0.001$ & 0.64 \\
\hline & $\left(\mathrm{Do}^{2} \times \mathrm{H}\right)\left(\mathrm{cm}^{2} \mathrm{~m}\right)$ & $\ln (y)=0.7206 \times \ln (x)-3.628$ & $<0.001$ & 0.77 \\
\hline & $\mathrm{H}(\mathrm{m})$ & $\ln (y)=2.2275 \times \ln (x)-4.043$ & $<0.001$ & 0.80 \\
\hline \multicolumn{5}{|c|}{20 years old secondary forest } \\
\hline \multirow[t]{3}{*}{ Leaf dry biomass (kg) } & Do $(\mathrm{cm})$ & $\ln (y)=2.0957 \times \ln (x)-4.559$ & $<0.001$ & 0.63 \\
\hline & $\left(\mathrm{Do}^{2} \times \mathrm{H}\right)\left(\mathrm{cm}^{2} \mathrm{~m}\right)$ & $\ln (y)=0.7598 \times \ln (x)-4.752$ & $<0.001$ & 0.70 \\
\hline & $\mathrm{H}(\mathrm{m})$ & $\ln (y)=1.9968 \times \ln (x)-4.939$ & $<0.001$ & 0.64 \\
\hline \multirow[t]{3}{*}{ Branch dry biomass (kg) } & Do $(\mathrm{cm})$ & $\ln (y)=2.5308 \times \ln (x)-5.047$ & $<0.001$ & 0.77 \\
\hline & $\left(\mathrm{Do}^{2} \times \mathrm{H}\right)\left(\mathrm{cm}^{2} \mathrm{~m}\right)$ & $\ln (y)=0.8783 \times \ln (x)-5.254$ & $<0.001$ & 0.86 \\
\hline & $\mathrm{H}(\mathrm{m})$ & $y=0.003(x)^{0.9181}$ & $<0.001$ & 0.75 \\
\hline \multirow[t]{3}{*}{ Stem dry biomass $(\mathrm{kg})$} & Do $(\mathrm{cm})$ & $\ln (y)=2.3751 \times \ln (x)-4.039$ & $<0.001$ & 0.82 \\
\hline & $\left(\mathrm{Do}^{2} \times \mathrm{H}\right)\left(\mathrm{cm}^{2} \mathrm{~m}\right)$ & $\ln (y)=0.8450 \times \ln (x)-4.244$ & $<0.001$ & 0.88 \\
\hline & $\mathrm{H}(\mathrm{m})$ & $\ln (y)=2.1410 \times \ln (x)-4.419$ & $<0.001$ & 0.75 \\
\hline \multirow[t]{3}{*}{ Aboveground biomass (kg) } & Do $(\mathrm{cm})$ & $\ln (y)=2.4014 \times \ln (x)-3.411$ & $<0.001$ & 0.83 \\
\hline & $\left(\mathrm{Do}^{2} \times \mathrm{H}\right)\left(\mathrm{cm}^{2} \mathrm{~m}\right)$ & $\ln (y)=0.8571 \times \ln (x)-3.621$ & $<0.001$ & 0.90 \\
\hline & $\mathrm{H}(\mathrm{m})$ & $y=0.008(x)^{1.1279}$ & $<0.001$ & 0.77 \\
\hline
\end{tabular}

Note: $P$ values of the regression analysis are shown. Adjusted $R^{2}$ denotes multiple coefficients of determination. 


\section{REFERENCES}

[1] M.C. Ruiz-Jaen, C. Potvin, Tree diversity explains variation in ecosystem function in a neotropical forest in Panama, Biotropica, 42(6), 2010, pp. 638646.

[2] K. Hairiah, S.M. Sitompul, M. Van Noordwijk, C.A. Palm, Methods for Sampling Carbon Stocks Above and Below Ground, ASB Lecture Note 4B, International Centre for Research in Agroforestry, Bogor, 2001, 23 pp

[3] Q.M. Ketterings, R. Coe, M. Van Noordwijk, Y. Ambagau, C.A. Palm, Reducing uncertainty in the use of allometric biomass equations for predicting aboveground tree biomass in mixed secondary forests, Forest Ecology and Management, 146, 2001, pp. 199-209.

[4] J.E. Brower, J.H. Zar, C.N. Von Ende, Field and Laboratory Methods for General Ecology, 3rd Ed, Wm. C. Brown Publishers, USA, 1990, 237 pp.

[5] K. MacDicken, A Guide to Monitoring Carbon Storage in Forestry and Agroforestry Projects, Winrock International, USA, 1997.

[6] T.M. Basuki, P.E. Van Laake, A.K. Skidmore, Y.A. Hussin, Allometric equations for estimating the aboveground biomass in tropical lowland dipterocarp forest, Forest Ecology and Management 257 pp. 1684-1694

[7] P.S. Curtis, Estimating Aboveground Carbon in Live and Standing Dead Trees, in: C.M. Hoover (Ed.), Field Measurements for Forest Carbon Monitoring: a Landscape-Scale Approach, Springer, USA, 2008, pp. 39-44.

[8] I. Heriansyah, N.M. Heriyanto, C.A. Siregar, Demonstration Study on Carbon Fixing Forest Management in Indonesia, in: Proceeding Climate Change, Forests and Peatlands in Indonesia, Series 1, Wetlands International, Canada, 2002.

[9] Ministry of Forestry Indonesia, Development of Allometric Equations for Estimating Forest Carbon Stocks Based on Field Measurement (Ground Based Forest Carbon Accounting), Centre for Standardization and Environment, Ministry of Forestry, Indonesia, 2011, 6 pp.

[10] J. Chave, C. Andalo, S. Brown, M.A. Cairns, J.Q. Chambers, D. Eamus, F.H. Fölster, F. Fromard, N. Higuchi, T. Kira, J.P. Lescure, B.W. Nelson, H. Ogawa, H. Puig, B. Riera, T. Yamakura, Tree allometry and improved estimation of carbon stocks and balance in tropical forests, Oecologia, 145, 2005, pp. 87-99.
[11] Karyati, I.B. Ipor, I. Jusoh, M.E. Wasli, I.A. Seman, Composition and diversity of plant seedlings and saplings at early secondary succession of fallow lands in Sabal, Sarawak, Acta Biologica Malaysiana, 2(3), 2013, pp. 85-94.

[12] Karyati, I.B. Ipor, I. Jusoh, M.E. Wasli, Soil properties under various stages of secondary forests at Sarawak, East Malaysia, J Trop For Environ, 4(1), 2014, pp. 28-39.

[13] Karyati, I.B. Ipor, I. Jusoh, M.E. Wasli, Tree stand floristic dynamics in secondary forests of different ages in Sarawak, Malaysia, Biodiversitas, 19(3), 2018, pp. 687-693.

[14] K. Hairiah, S. Rahayu, Pengukuran "Karbon Tersimpan" di Berbagai Macam Penggunaan Lahan, World Agroforestry Centre-ICRAF, Bogor, 2007, pp. 29-55.

[15] T. Hashimoto, T. Tange, M. Masumori, H. Yagi, S. Sasaki S, K. Kojima, Allometric equations for pioneer tree species and estimation of the aboveground biomass of a tropical secondary forest in East Kalimantan, Tropics, 14, 2004, pp. 123130.

[16] T. Kenzo, T. Ichie, D. Hattori, T. Itioka, C. Handa, T. Ohkubo, J.J. Kendawang, M. Nakamura, M. Sakaguchi, N. Takahashi, M. Okamoto, A. TanakaOda, K. Sakurai, I. Ninomiya, Development of allometric relationships for accurate estimation of above- and below-ground biomass in tropical secondary forests in Sarawak, Malaysia Journal of Tropical Ecology, 25, 2009, pp. 371-386.

[17] Y. Kiyono, Hastaniah, Pattern of slash-and-burn land use and their effects on forest succession: Swidden-land forests, in: Borneo Bulletin of the Forestry and Forest Products Research Institute, 4, 2005, pp. 259-282.

[18] C.A. Sierra, J.I. Del Valle, S.A. Orrego, F.H. Moreno, M.A. Harmon, M. Zapata, G.J. Colorado, M.A. Herrera, W. Lara, D.E. Restrepo, L.M. Berrouet, L.M. Loaiza, J.F. Benjumea, Total carbon stocks in a tropical forest landscape of the Porce Region, Colombia Forest Ecology and Management, 243, 2007, pp. 299-309.

[19] R.S. Capers, R.L. Chazdon, A.R. Brenes, B.V. Alvarado, Successional dynamics of woody seedling communities in wet tropical secondary forests, Journal of Ecology, 93, 2005, pp 10711084.

[20] E. Romell, G. Hallsby, A. Karlsson, C. Garcia, Artificial canopy gaps in a Macaranga spp. dominated secondary tropical rain forest-effects on 
survival and above ground increment of four under-planted dipterocarp species, Forest Ecology and Management 255, 2008, pp 1452-1460.

[21] J. G. Saldarriaga, D.C. West, M.L. Tharp, C. Uhl, Long-term chronosequence of forest succession in the upper Rio Negro of Colombia and Venezuela, Journal of Ecology, 76, 1988, pp. 938-958 